Portland State University

PDXScholar

$5-23-2019$

\title{
Brand Logo Shape and Brand Personality
}

Kyle P. Gold

Portland State University

Follow this and additional works at: https://pdxscholar.library.pdx.edu/honorstheses

Let us know how access to this document benefits you.

\section{Recommended Citation}

Gold, Kyle P., "Brand Logo Shape and Brand Personality" (2019). University Honors Theses. Paper 713. https://doi.org/10.15760/honors.730

This Thesis is brought to you for free and open access. It has been accepted for inclusion in University Honors Theses by an authorized administrator of PDXScholar. Please contact us if we can make this document more accessible: pdxscholar@pdx.edu. 


\title{
Brand Logo Shape and Brand Personality
}

\section{by}

\section{Kyle Gold}

An undergraduate honors thesis submitted in partial fulfillment of the

\author{
requirements for the degree of \\ Bachelor of Science \\ In \\ University Honors \\ and \\ Marketing
}

\begin{abstract}
Thesis Adviser
Jacob Suher - Assistant Professor of Marketing Ph.D.
\end{abstract}

Portland State University 


\begin{abstract}
Brand logos are a critical element of brand image equity in the process of visually conveying brand values to a consumer base. While previous studies have discussed logo design elements like color, typography, and complexity on their ability to convey brand attributes - little research has been done on logo shape. This study examines the relationship between brand logo shape - that of the geometric outline of a logo - and the concept of brand personality. To accomplish this, stimuli was created and presented via survey to test if consumers could recognize brand personality factors from a series of designed geometric brand logos in order to find distinct relationships between brand personality factors and individual geometric shapes. While the results were inconclusive on this proposed relationship overall, this study mainly supported previous marketing literature on brand logo angularity vs. roundness on brand personality factors of excitement and ruggedness.
\end{abstract}

\title{
Introduction
}

Brand logos, no matter how simple or complex, tend to be associated as the symbolic “face" of a company (Farhana 2012, Klink 2003, Park et al. 2013), and some companies have invested accordingly. For the purposes of this study, a "brand logo" is a mark, symbol, or image that is used to brand a company / product for the purposes of identification and recognition. In 2008, Pepsi spent roughly five months and upwards of $\$ 1$ million to redesign the logo so that the inner curves of the logo form "smiles" (Appendix A). This was to create a logo that Pepsi's VP portfolio manager described as “...dynamic and more alive” (Zmuda 2008). Whether the logo's design effectively symbolizes what the VP described is subjective, but Pepsi felt the need to strengthen their brand identity through a revitalization of their brand logo and to better convey desired consumer perceptions to their target market. This case is not unusual, as market research 
has shown the importance of strong brand image equity for a company (Faircloth et al. 2001, Park et al. 1986). Brands with market power are better able to compete in price-flexibility against less established brands due to overall positive consumer associations and assumptions of the brand and its respective product line (Kay 2006). Brands with positive consumer associations of brand identity have also been shown to increase product or service purchase intent and brand loyalty (Cobb-Walgren et al. 1995).

Once a brand is established in the market, brand image is critical for market expansion. Strong brand associations garner larger brand affect - leading to similar brand affect for new products introduced into the brand family (Broniarczyk \& Alba 1994). Recognizable brands established by effective advertisement and placement also reap the benefits of increased customer preference of their products and overall brand loyalty (Hoeffler \& Lane 2003). However, once a brand has been established in the market, a significant change to brand image or aesthetic has been shown to negatively impact brand equity through lower brand evaluations and reduced brand recognition (Walsh et al. 2010; Walsh 2005). With the importance of brand identity / image in competing with other brands, thorough initial design and implementation of brand elements is key to creating strong brand equity.

While every major company has a brand logo, questions of effective logo design arise in the field of marketing, advertising, and graphic design on what makes a logo effective in visually conveying brand information to the consumer base. To answer this question, it is critical to know what the purpose of a company logo is, beyond the visual branding of a product or company. For many, a brand logo is the first visual introduction between a company and consumer (Henderson \& Cote 1998; Foroudi et al. 2014) and represents the company in some shape or form. In 2000, BP aimed to change viewers perceptions on their company with a complete brand redesign, with 
their new "Helios" logo in the forefront designed to represent the sun and nature (Appendix B). (Landor 2018, Farhana 2013). Beyond representation, a logo is generally symbolic in nature (Henderson \& Cote 1998; Kilin 2003; Adir et al. 2012) and is not only used to "brand the brand" but as a method of conveying the company image to the consumer base. Looking at the BP example again, while the logo may represent the sun and nature, it can be argued that this representation of the elements is for the purpose of visually conveying BP's organizational change towards sustainability and green practices.

With this idea that brand logo design has an impact on the relationship between consumer and brand, questions have been posed on how an effective logo can be created in order to promote a desirable brand image. In their research Henderson and Cote define a "good" logo as one that "... [is] recognizable, familiar, elicit a consensually held meaning, and evoke positive effect". While some logos can garner brand loyalty and likability through market leadership (Foroudi et al. 2014), execution of design elements, focused on producing aesthetically engaging logos for their target demographic, has been shown to create brand loyalty (Walsh 2005, Klink 2003, Foroudi 2014, Müller et al. 2013) and brand liking (Pittard 2014, Müller et al. 2013). This study specifically has looked at the brand logo design element of shape and the effectiveness of that element in conveying brand personality to consumers. The following section underlines key research on brand logos and brand personality before developing a preliminary hypothesis related to logo shape.

\section{Conceptual Background}

\section{Brand Logos and Brand Personality}

With a brand, companies also aim for their brand identity to be desirable and have a positive effect on their consumers. A recognized understanding of desirable consumer brand 
perceptions is discussed in Jennifer Aaker's, "Dimensions of Brand Personality," which defines brand personality, "...the set of human characteristics associated with a brand" (1997). These characteristics were later defined by 42 personality traits of a brand which are separated by "the Big Five" overarching factors in brand personality: Sincerity, Excitement, Competence, Sophistication, and Ruggedness (Aaker 1997). Aaker theorized that these effective utilizations of brand facets could have lasting influence on consumer preference / brand liking in regard to a brand's associated personality. Research into these effects of brand personality have shown multiple benefits in understanding the effect it has on consumer preferences. Desirable brand personalities elicit a positive effect on brand attractiveness (Kim et al. 2001, Hayes et al. 2006), purchase intention, strategy for brand promotion and organization (Freling et al. 2011), and customer brand impression (Fennis \& Pruyn 2006).

These theorized effects have been implemented in controlled experiments and by organizations with varying degrees of success. Specifically, Aaker's concepts of brand personality have been widely used in understanding phenomena of customer-brand relationships and brand equity. Extensions of Aaker's brand personality theory into destination personality theory discovered that individuals ascribe personality to locations, to which tourism industries can develop images to correlate with assumed location personality (Ekinci \& Hosany 2006, Aguilar et al. 2014). Brands that utilize brand personality effectively also generate brand attachments based on emotional loyalty of customers who feel the brand's personality best represents their ideal self or perceived positive attributes (Malär 2007). Additionally, a constant component of understanding the process of conveying brand personality to consumers is that of the correlation between brand and brand design. One factor of brand design that has received much attention is that of a brand logo. Research into the design of brand logos has found that 
careful consideration and execution of elements like color (Foroudi et al. 2014; Henderson \& Cote 1998; Labrecque \& Milne 2012; Müller, et al. 2013; Klink 2003), complexity (Henderson \& Cote 1998; Grinsven \& Das 2014; Luffarelli et al. 2019.) and shape (Walsh 2005; Jiang et al. 2016; Pittard et al. 2007; Luffarelli et al. 2019.) have positive effects on consumer brand recognition, brand liking, and brand personality. To understand the hypothesized relationship between brand logo shape and brand personality, the following section discusses element of logo shape in the field of design and market research.

\section{Logo Shape}

While studies have looked at elements like complexity (Grinsven \& Das 2014; Luffarelli et al. 2019.) and color (Foroudi et al. 2014; Henderson \& Cote 1998; Labrecque \& Milne 2012; Müller et al. 2013; Klink 2003) in logo design to create desirable brand image equity, empirical research into the shape of a brand logo in this aspect has been minimal, most likely due to the subjective nature of what makes "good" design. Shape is defined in the Merriam Webster dictionary as, "the visible makeup characteristic of a particular item or kind of item" which can be narrowed to "a standard or universally recognized spatial form." For logo design, the shape of a brand logo can be considered the structural elements - like length and symmetry - or the geometric elements - like angular or round - that make up the spatial form of the logo. An example would be the "natural" representative shape of an "apple" in the Apple logo (Appendix C). However, the Apple logo has many more aspects of shape, like its asymmetry (the "bite" out of the apple) and its rounded edges. On the opposite spectrum, but in the same industry, the Microsoft Windows logo (Appendix D) would be considered more abstract as a representation of a window, but its shape is horizontally and vertically symmetrical - with no round edges. Research into aesthetically pleasing shapes in logo design and proper implementation has created 
generally accepted guidelines for logo design. One of the most influential is the "Guidelines for Selecting or Modifying Logos," which defines "good" logo design by 13 design characteristics. In these 13 characteristics, shape is a key factor in multiple categories, like "round", "symmetry", and "balance" (Henderson \& Cote 1998). Further studies using these established principles have also discussed logo shape in their positive role in brand loyalty (Walsh 2005) and brand attitudes (Luffarelli et al. 2019).

Additionally, logo shape has shown to have some amount of impact regarding consumer brand recognition. This research, however, mostly focuses on angularity and roundness of logo shape in regard to logo design, and the stimuli produced when presented to consumers (Walsh 2005; Jiang et al. 2016; Pittard. et al. 2007). This idea has been developed into more detailed analysis of shape design elements in recent years, distilling shape into concepts of movement and asymmetry in geometric logo shape to elicit stimuli of exciting brand personality (Luffarelli et al. 2019). However, further research is needed on overarching logo shapes, specifically that of the general spatial outline of a logo, and its impact on logo design perceptions. While this idea is inherently tied to design elements of complexity, angularity, and roundness; the independent variable described by "Guidelines for Selecting or Modifying Logos" pertaining to this idea is that of geometric designs - logo designs that are abstract and synthetic in appearance. Geometric shaped logos have been found to be visually less meaningful, as more abstraction leads to decreased viewer understanding of purposeful design especially in relation to logos (Dondis 1973). However, when compared with previous studies of logo design pertaining to that of logo angularity / roundness, shape complexity, and symbolism - all elements that compose the general outline of a logo shape; research on geometric logo shape can encompass these elements for 
more general discussion of brand logo design, including that of its effects on brand personality (Grohmann 2008).

\section{Visuospatial relations and Geometric Shapes}

In this study, the focus is on logos with the primary spatial outline of geometric shapes in order to find connections between basic elements of logo shape and brand personality. Regarding the definition given of "shape" pertaining to logo design, research focuses on the geometric structure of logos. Geometric shapes tend to produce visual stimuli when initially viewed, which can be considered a form of visuospatial working memory (Jiang et al. 2016). Visuospatial working memory is the mental process of short-term association of shapes between one another and using this information to derive new form. An example of this would be seeing a circle and associating it with the roundness of a sphere or ball. By applying this logic to logo shape, it could be assumed that the shape of an object has implicit meaning when initially viewed. Research into this phenomenon, specifically on geometric shapes is a subject of debate in fields such as anthropology, psychology, and graphic design. While most of these assumed representations are based off speculation of spatial recognition similar to Gestalt theory, some research has validated that there is a possible correlation between geometric shapes and human characteristics (Adir et al. 2012; Larson et al. 2011; Aronoff 2006). Geometric shapes in relation to human thought have evoked meaning and passive cognitive relationships. These emotions are specifically tied to human recognition of angular and circular shapes. Recognition of angular shapes throughout multiple western and non-western cultures have been revealed to elicit negative stimuli, that of unease, excitement, and anger (Larson et al. 2011; Aronoff 2006). Triangles, and their specific orientation, were generally seen to represent stressed or irritated facial structures while circular shapes conveyed calm and collected human facial structures (Aronoff 2006). Cognitive 
relationships to touch have also been revealed in associations of shapes, with a tendency for people to prefer rounded/curved visual objects over that of angular (Bar \& Maitel, 2006;

Westerman et al. 2012). Simple geometric shapes and their orientations when presented have been shown to hold symbolic meaning for cultures as well. Downward facing "Vs" were found to be more unpleasant and foreboding when recognized in distressing images, but the opposite effect with upward facing "Vs" and circles - which were associated with pleasant and comforting emotions (Larson et al. 2011; Aronoff 2006). Applied to product marketing, angular shapes tend to convey consumer assumptions of product "hardness" and rounded shapes convey "softness" (Jiang et al. 2016; Westerman et al. 2012). This concept of visual imagery of imagined touch and feel of an object relates to the concept of haptic imagery, the mental recognition / construction of touch (Peck \& Barger 2009). This idea is key to understanding the deconstruction of shapes in relation to working memory, as positive and negative "feel" of certain shapes (like sharpness and roundness) can influence consumer intention (Peck \& Barger 2009). Furthermore, breaking down the elements of "hardness and softness" have revealed facets relating to assumed product durability and comfort respectively when applied to logo shape (Jiang et al. 2016).

Prominent opinions of the implicit meaning of basic geometric shapes are from graphic designers (Arhipova 2018; Bhavya 2016; Christie 2017; Macnab 2015; Pahwa 2018; The Logo Company 2018; Tailor Brands 2018). While differences in opinions of what geometric shapes imply psychologically when viewed, there are many similarities in assumptions. Three distinct geometric shapes discussed by these designers are circles, squares, and triangles. Circles are said to represent harmony and softness, squares represent balance and security, and triangles represent movement and intelligence. Designers have also argued that when brands design their brand logos around these geometric shapes, they are able to implicitly suggest the emotions to 
their customer base. However, it is important to note that these discussions are highly qualitative in nature, and speculation on validity and false correlation in these assumptions is a valid basis for debate.

\section{Research Question}

With elements like color theory intertwined with human emotions and perceptions (Labrecque \& Milne 2012), could the same principles be applied with manipulated geometric shapes when applied to logo design for the purposes of influencing brand personality? Brand personality factors are based on human characteristics, many of which are similar to associations of shape with psychology. With this understanding, this research aims to find a correlation between these two phenomena of human characteristics associated with brand imagery in order to find significant data that suggests a certain geometric shape in logo design can be used to promote positive recognition of certain facets of the brand personality. Based on prior research on brand personality, logo design, and the correlation between geometric shapes and human emotions, this study's preliminary and overarching goal is to find whether the spatial outline of a logo with certain geometric shape can elicit consumer recognition of specific facets of brand personality.

\section{Logo Shape Hypotheses}

In order to test for a correlation between geometric logo shape and brand personality a set of brand logos with a primary outline of a geometric shape (square, circle, and triangle) were created in order to find relationships to three factors of five factors in the brand personality model (Appendix E, F, and G respectively). The first shape, square, in regard to graphic design is qualitatively described as a shape of stability, reliability, and technological (Arhipova 2018; Bhavya 2016; Christie 2017; Macnab 2015; Pahwa 2018; The Logo Company 2018; Tailor 
Brands 2018). A basic geometric square is symmetrical both horizontally and vertically, and this symmetry leads to positive viewer evaluations of design due to pattern recognition (Henderson \& Cote 1998). Symbolically, squares in design have been theorized to represent a "boundaried property," that of a floor or wall (Frutiger \& Andrew 1998). These ideas have been discussed in relationship to street signs, as symmetrical shapes (such as squares and triangles) used as backgrounds help establish visually recognizable “order” (Pettersson 1999). Applying these ideas in corporate logo design, squares logos have been theorized to influence consumer perceptions on brand stability (Adîr et al. 2012), and product reliability / durability (Jiang et al. 2016). With both the qualitative and empirical research on squares, and the visual perceptions of traits like stability and order relating to the brand personality traits of the brand personality factor "competence", this study theorizes that:

H1a: Compared to other shapes, a logo with a geometric outline shape of "square" will be perceived as more competent in relationship to brand personality.

The second shape of study, the circle, is described qualitatively as a shape of unity, kindness, and harmony (Arhipova 2018; Bhavya 2016; Christie 2017; Macnab 2015; Pahwa 2018; The Logo Company 2018; Tailor Brands 2018). Circles are primarily known for its positive symbolism and viewer perceptions due to its rounded element. A perfect circle is also symmetrical, which convokes positive affect in consumer perceptions (Henderson \& Cote 1998). As previously discussed, rounded shapes when used as an icon or logo conveys the haptic imagery of "softness" due to the circles geometric lack of any straight lines (Jiang et al. 2016; Larson et al. 2011; Aronoff 2006). This "softness" of shape conveys positive / alluring mental stimuli due to the inherent relationship between circles and safety / comfort - which is theorized to be more inviting then angular shapes (Larson et al. 2011; Aronoff 2006; Pettersson 1999). 
Additionally, circle logos have been interpreted to influence consumer perceptions of brand balance (Adîr et al. 2012), product comfort (Jiang et al. 2016), and less exciting when compared to asymmetric shapes (Luffarelli et al. 2019). Research specifically on brand personality and logo design also found a small correlation between logo roundness and positive customer perceptions of brand sincerity (Grohmann 2008). With both the qualitative and empirical research on circles, and the visual perceptions of traits like comfort and softness relating to the brand personality traits of the brand personality factor "sincerity", this study theorizes that:

H1b: Compared to other shapes, a logo with a geometric outline shape of "circle" will be perceived as more sincere in relationship to brand personality.

The final geometric logo shape of study is the triangle, described qualitatively as a shape of power, intelligence, and directive (Arhipova 2018; Bhavya 2016; Christie 2017; Macnab 2015; Pahwa 2018; The Logo Company 2018; Tailor Brands 2018). As a geometric shape, triangles are somewhat symmetrical with overall asymmetry (Henderson \& Cote 1998). Triangles are also primarily angular with their outline forming three distinct points. These angular points not only elicit consumer perceptions of excitment (Luffarelli et al. 2019) and durability (Jiang et al. 2016), but that of danger. Triangles as symbols have been found to correlate with emotions of fear and power, due to Gestalt theory ideas of cognitive recognition of "sharp" objects relating to haptic visualization of pain (Larson et al. 2011; Aronoff 2006). However, when discussing triangles as symbols, it is important to note the direction to which the tip of the triangle (defined in this study as the direction of the discernable singular point in relationship to the other two points of an equilateral triangle) is facing when presented as an icon. As previously discussed, a downward facing "V" has been found to create negative connotations while an upward facing "V" invokes more positive connotations (Larson et al. 
2011). This idea of danger is applied to road sign design as well, as geometric triangles have been theorized to best convey warning or directive guidance (Frutiger \& Andrew 1998).

Additionally, when applied to logo design, triangles convey brand urgency (Adîr et al. 2012) and excitement (Luffarelli et al. 2019). With both the qualitative and empirical research on triangles, and the visual perceptions of traits like excitement and direction relating to the brand personality traits of the brand personality factor "exciting", this study theorizes that:

H1c: Compared to other shapes, A logo with a geometric outline shape of "triangle" will be perceived as more exciting in relationship to brand personality.

\section{Tests \& Stimuli}

To test our primary hypothesis - that brand logos with a spatial outline of a certain geometric shape can elicit consumer recognition of specific facets of brand personality - a survey with created geometric brand logos was developed. All brand logos were created using Adobe Illustrator CC 2019 for digital display in black and white. Logos were designed to be low recognition, low "naturalness," and high affect based on Henderson \& Cote's 1998 "Guidelines for Selecting and Modifying Logos" general description of a "low-investment logo." While a low-investment logo is described to have false recognition based on existing brand logos, the designed geometric logo shapes for this survey were created with the intention of no false recognition of existing brand logos in order to isolate logo shape as the main driver for brand personality interpretation. In regards to low "naturalness," since the basis for testing only geometric shapes relies on the symbolic nature of shapes, low "naturalness" for the purposes of this study is to "not represent anything distinctly natural" (like a combination of circles that look like an eye or a triangle shaped to look like a Mt. Fuji), and the spatial outline of the logos were created to not directly depict a distinct object (like an arrow or a box). Each logo was created 
using a single constant geometric shape, with the primary outline being that of a square (Appendix E), circle (Appendix F), and equilateral triangle (Appendix G) respectively. Additionally, in order to not simply present a geometric shape as a logo, smaller versions of the geometric shapes were included in the logo as a distinct asymmetric element of the logo design. This asymmetry was included to increase perceived design affect of the logos (Henderson \& Cote 1998; Luffarelli et al. 2019), as presenting geometric shapes as logos was assumed to be unable to elicit any participant interest. Additionally, an alternative version of each logo was created by horizontally flipping the image in order to test for any possible testing bias based on undesirable false recognition of a logo design.

The survey then presented these created geometric brand logos in a digital survey format. The survey was distributed to 85 undergraduate students, receiving extra credit in their respective business class for participating. The survey was created using Qualtrics, and was distributed digitally to participants, who were able to take the test without proctoring. Altogether, 31 Male participants and 54 female participants were surveyed, with a variety of ages $\left(\mathrm{M}_{\mathrm{age}}=25, \mathrm{SD}_{\mathrm{age}}=\right.$ 5.65, $\operatorname{Min}_{\mathrm{age}}=19, \mathrm{Max}_{\mathrm{age}}=47$ ), participants were briefed on the Jennifer Aaker's concept of brand personality and were asked to evaluate the provided logos based on their interpretations of perceived personality. Three logos of a possible six (three geometric logos with a respective alternate), each pertaining to a certain geometric shape, were presented in a random order to participants. Participants were first asked to rate overall affect of the given logo on multiple fivepoint likert scales, based on the questions used in "Guidelines for Selecting and Modifying Logos" to test for logo effect (interesting, high quality, good, distinctive, and likability). Participants were then presented with the 15 brand personality facets, and asked to choose three from the list which best describes the brand personality of the presented logo. Finally, 
participants were asked to evaluate all 15 brand personality facets regarding how well it describes the logo on a five-point semantic scale. This process was then repeated for three logo variations, one for each geometric logo shape, and concluded with a self-evaluation of the participant's own perceived personality. Once results were collected, data was downloaded and analyzed with Microsoft Excel 2016 and SAS. Overall relationship between the logos and brand personality were found with correlation analysis to determine whether the results were above a standard threshold for a statistically significant relationship $(\approx|\mathrm{r} \geq 0.2|, p<0.05)$. Shapes presented were coded with dummy variables for each of the three possible shapes, with a one signifying that the participant was shown a specific shape and zeros for the shapes that were not shown at that time.

\section{Results}

\section{Square Affect and Recognition}

For H1a (Square), the brand logos were overall seen as low affect, due to the generally low perceptions of the logos in every category $\left(\mathrm{r}_{\text {affect }}=-0.32, p<0.001\right)$. For the results discussion for each shape, affect evaluations were found to be highly correlated, and are reported based on a grouped correlation coefficient (Appendix H). For recognition, 96.7\% of 85 participants did not falsely recognize the logo, $92.9 \%$ felt that that the logo did not strongly resemble another logo they have seen before, and $74.1 \%$ of participants felt that the logo is not similar to a logo they have seen before. Overall, this study classifies overall recognition of the logo to be low recognition.

\section{Square Personality}

For the analysis of hypothesis H1a we conducted a correlation analysis of each brand facet in relationship to the presented square logo. For analysis of relationships between square 
logos presented and brand personality facets, no significant relationships were found between any of the three facets related to competence; reliable $\left(\mathrm{r}_{\text {reliable }}=0.060, p=0.34\right)$, intelligent $\left(\mathrm{r}_{\text {intelligent }}=0.094, p=0.135\right)$, and successful $\left(\mathrm{r}_{\text {successful }}=0.039, p=0.533\right)$. Additionally, no significant positive relationships between any of the brand personality and square logo facets were discovered in the data. Significant negative relationships, however, with brand personality facets were found with square logos including wholesome $\left(\mathrm{r}_{\text {wholesome }}=-0.200, p=0.002\right)$, cheerful $\left(\mathrm{r}_{\text {cheerful }}=-0.243, p<0.001\right)$, daring $\left(\mathrm{r}_{\text {daring }}=-0.208, p=0.001\right)$, imaginative $\left(\mathrm{r}_{\text {imaginative }}=\right.$ $-0.237, p<0.001)$, charming $\left(\mathrm{r}_{\text {charming }}=-0.200, p=0.001\right)$, and outdoorsy $\left(\mathrm{r}_{\text {outdoorsy }}=-0.279, p<\right.$ $0.001)$.

\section{Square Discussion}

Based on this study's analysis, we can conclude that the there is no relationship between the geometric square logo shape and the brand personality factor competence. However, while no significant positive relationships were found, the significant negative relationships between the square logos and brand personality facets support previous studies. Negative relationships in three of the four facets related to excitement were found in similar research discussing the effects of asymmetrical logos and excitement. Logos that were symmetrical were overall less exciting regarding brand personality then logos that were asymmetrical (Luffarelli et al. 2019). While only a single facet of sincerity, cheerful, showed a somewhat significant negative relationship with square logos, this analysis is supported by comparisons of round / angular logos and brand personality - with angular logos eliciting lower consumer perceptions of product softness (Jiang et al. 2016). Additionally, overall negative evaluations of logo affect for square logos is also supported by previous research. Consumers positively evaluated rounded logos in design while angular logos were evaluated lower, especially with brands exhibiting more sincere brand 
personality traits (Jiang et al. 2016; Walsh 2005; Westerman et al. 2012). Additionally, overall negative evaluations of logo affect may have resulted in overall lower evaluations of brand personality due to general dislike of the logo presented.

\section{Circle Affect and Recognition}

For hypothesis $\mathrm{H} 1 \mathrm{~b}$ the brand logos were overall seen as medium affect $\left(\mathrm{r}_{\mathrm{affect}}=0.090, p\right.$ $=0.151$ ), due to the generally impartial perceptions of the logo design. For recognition, $98.8 \%$ of 85 participants did not falsely recognize the logo, $84.7 \%$ felt that that the logo did not strongly resemble another logo they have seen before, and $72.9 \%$ of participants felt that the logo is not similar to a logo they have seen before. Overall, this study classifies overall recognition of the logo to be low recognition.

\section{Circle Personality}

For the analysis of hypothesis $\mathrm{H} 1 \mathrm{~b}$ we conducted a correlation analysis of each brand facet in relationship to the presented circle logo. For analysis of relationships between circle logos presented and brand personality facets, two positive relationships were found between the two of the four facets related to sincerity; down-to-earth $\left(\mathrm{r}_{\mathrm{down}}\right.$-to-earth $\left.=0.000, p=1\right)$, honest $\left(\mathrm{r}_{\text {honest }}=0.075, p=0.23\right)$, wholesome $\left(\mathrm{r}_{\text {wholesome }}=0.183, p=0.003\right)$, and cheerful $\left(\mathrm{r}_{\text {cheerful }}=0.191\right.$, $p=0.002)$. A significant relationship was found between circle logos and brand personality facet, imaginative ( $\left.\mathrm{r}_{\text {imaginative }}=0.218, p=0.001\right)$, a facet under the factor excitement. Additionally, one significant negative relationship with brand personality facet, tough, was associated with circle $\log 0 \mathrm{~s}\left(\mathrm{r}_{\mathrm{tough}}=-0.320, p<0.001\right)$.

\section{Circle Discussion}

While there is a small correlation between a portion of the brand personality factor, sincerity, and the circle logos presented, this study finds that correlation too minimal to make 
any large claims. However, the negative relationship between the brand personality facet, tough, does provide additional support for previous research. With the circle logos presented as the only logos of the group with a predominantly "round" shape with no hard edges, previous research specifically relates rounded brand logos to be perceived as softer (Jiang et al. 2016; Walsh 2005; Westerman et al. 2012), more sincere (Grohmann 2008), and unrelated to the brand factor of ruggedness (Jiang et al. 2016). Overall, this study found minimal significant correlations between any other variables in the experiment and moderate affect for the logo overall (Appendix I).

\section{Triangle Affect and Recognition}

For hypothesis H1c, the brand logos were overall seen as medium affect, due to the generally positive evaluations of the triangle compared to other shapes $\left(\mathrm{r}_{\text {affect }}=0.231, \mathrm{p}<0.001\right)$. For recognition, $91.8 \%$ of 85 participants did not falsely recognize the logo, $78.8 \%$ felt that that the logo did not strongly resemble another logo they have seen before, and $55.5 \%$ of participants felt that the logo is not similar to a logo they have seen before. Overall, this study classifies overall recognition of the logo to be low recognition, with a medium recognition of similarity between the logo and existing brand logos.

\section{Triangle Personality}

For the analysis of hypothesis H1c we conducted a correlation analysis of each brand facet in relationship to the presented circle logo. For analysis of relationships between triangle logos presented and brand personality facets, two significant relationships were found between the four facets related to excitement; daring $\left(\mathrm{r}_{\text {daring }}=0.244, p<0.001\right)$, spirited $\left(\mathrm{r}_{\text {spirited }}=0.163, p\right.$ $=0.001)$, imaginative $\left(r_{\text {imaginative }}=0.020, p=0.760\right)$, and up-to-date $\left(r_{\text {up-to-date }}=0.050, p=0.429\right)$. 
In addition, significant positive relationships between the both brand facets related to rugged were present as well; outdoorsy ( $\left.\mathrm{r}_{\text {outdoorsy }}=0.472, \mathrm{p}<0.001\right)$, and tough $\left(\mathrm{r}_{\text {tough }}=0.320, \mathrm{p}<0.001\right)$.

\section{Triangle Discussion}

Based on the data, our hypothesis that triangle logos could visually communicate the brand personality factor, exciting, is also under supported by the data. While the brand personality facet, daring, was found to have modest relationship with the triangle logos, overall it is was not substantial enough to make any distinct claim of correlation, but a small correlation can be assumed. However, substantial evidence from the strong positive relationships between the triangle logos presented and the brand personality facets of outdoorsy and tough were discovered. This provides evidence of a relationship between triangle logos and the brand personality factor, ruggedness. Previous research supports this correlation, as angular logos were perceived by consumers to be more rugged in comparison to rounded logos (Jiang et al. 2016). Additionally, psychographic research links "sharp" shapes to that of feelings of danger (Larson et al. 2011; Aronoff 2006), which is in tangent with consumer perceptions of ruggedness in brands (Maehle et al. 2011). The triangle logos were also met with moderately higher evaluations of affect then those of the circle and square logos, which is supported by previous research of overall brand logo asymmetry positively affecting consumer perceptions of logos (Luffarelli et al. 2019).

However, with the moderate amount of false recognition, these results may be skewed towards ruggedness, due to the association with outdoor sports apparel. Out of the participants who found that the logo to be strongly or similar to another logo, all but one noted an outdoor or sports brand. Multiple participants even believed that the logo was an REI logo (REI is known for their outdoor and mountain apparel). 


\section{General Discussion}

\section{Investigation results}

This investigation into the possible ability of geometric brand logo design to convey certain factors of brand personality discovered some evidence of a relationship but was ultimately inconsistent. While some quantitative relationships were found, the hypothesis that the geometric spatial outline of a brand logo can convey brand personality does not have enough supporting correlation between brand personality factors and the logos created to make a definitive assumption. However, this investigation does add to marketing literature through its support of previous research.

With the logos presented, the consensus was that the logos were somewhat poor in design and likeability, with the triangle logos evaluated higher overall. Regarding Henderson and Cotes 1998 guidelines, this is consistent with the risk of negative consumer perceptions of logos low in naturalness, high in abstract, and with low false recognition. With Henderson and Cote's guidelines 1998, false recognition for logos with low naturalness is critical for simple brand logos to garner high affect. The triangle logos presented that were associated with false recognition, while undesirable for the purposes of this study, also achieved the highest evaluations of logo design and affect - consistent with the description of a successful lowinvestment logo (Henderson \& Cote 1998). On the opposite spectrum, the square logos had the lowest false recognition among logos presented, as well negative evaluations of affect. This supports Henderson and Cote's 1998 guidelines for creating low-investment logos, as well as the limitations of basic logos abilities to communicate brand equity (Grinsven \& Das 2014; Dondis 1973). 
Where this study best advances brand logo design practice is the implications of roundness and angularity in logo shape. This investigation saw significant relationships between angularity and consumer evaluations of brand personality (Appendix I). The triangle logos were found to have strong relationships with that of rugged personality facets, and the square logos negative relationships in conveying sincere personality facets. Circle logos presented also elicited strong negative assumptions of brand toughness. These findings are consistent with previous studies discussing the impact of consumer assumptions of brand equity when presented with angularity in logo design (Jiang et al. 2016; Pittard 2007).

This study also combats the more qualitative assumptions of logo design present in graphic design literature. While graphic designers have assumptions on the visual attributes of geometric shapes and the symbolic imagery that can be conveyed when used in logo design, this study finds that more substantial consideration of design elements are needed in order to boost consumer recognition of brand attributes. While designers (Arhipova 2018; Bhavya 2016; Christie 2017; Macnab 2015; Pahwa 2018; The Logo Company 2018; Tailor Brands 2018) and market researchers (Adîr et al. 2012) have argued that geometric shapes alone, like squares, circles, and triangles, can convey imagery of kindness, softness, power, etc. - this study has shown that these basic shapes alone have limitations in brand logo design. While design and psychology literature argue that there are correlations between cognitive responses to geometric shapes in terms of visuospatial recognition and haptic imagery, these studies focus more on perceptions of the structure of these geometric shapes rather than their independent ability as a symbol (similar to that of market literature on angularity, roundness, and form) (Peck \& Barger 2009, Pettersson 1999). While geometric shapes may be effective in visual communication in 
other fields of design and image design, this study cannot make the same claim for brand logo design.

\section{Managerial Implications}

While this study was non-conclusive on its hypotheses, there are still important findings for brand logo design. While little significant data was collected on specific geometric shapes and their ability to convey brand personality, the effectiveness of precise logo design is still supported by this data. Solely relying on geometric shapes to convey brand personality or communicating brand values is ill-advised based on these findings, but implementation of rounded and angular design to convey certain brand personality facets is supported. Logo designers should carefully consider using angular shapes when trying to convey exciting and rugged brand personality traits, and rounded shapes when trying to convey more sincere traits. This information is especially relevant to start-up organizations, or brands relatively new to the market. With research suggesting that complex logos are more difficult for consumers to recognize and remember when a brand is young or new (Grinsven \& Das 2014) - it would be beneficial for brand managers to consider effective implementation of basic design elements, in order to create memorable brand logos. Managers may also consider using more natural / symbolic imagery in their brand logos or using logo shape to accent their designs rather than

focus on them (Henderson \& Cote 1998). Overall, careful consideration of logo design can help brands better convey brand personality traits, which previous market research discussed and supported in this study have found to positively affect general brand equity.

\section{Limitations and Further Research}

While this study developed little evidence in supporting its hypotheses, improving the experimental design and further research may find stronger relationships between brand 
personality and geometric logo design. Based on the overall low affect of the logos, and an undesirable false recognition with the triangle logos, a pretest evaluating logo affect for the created logos in the experiment could reduce undesirable testing stimuli. Concern over the validity of the square data is present, as overall negative perceptions of affect may have skewed evaluations of the brand personality facets. These created logos could be presented to graphic designers / brand managers for affect and recognition evaluation, which would create a stronger basis for correlation analysis. With the scope of this project, and the time limitations of survey implementation and data analysis, this research could be expanded in size and scope. A larger survey size, and multiple surveys presented to a wider demographic may find more substantial data then covered in this preliminary study. Some participants also voiced confusion with the given brand personality facets names. Further statistical analysis for the purposes of discovering additional correlation between brand personality factors and logo design may have also revealed other significant relationships.

Future research into geometric logo designs, especially whether the spatial outline of the shape could prove valuable. Future experiments, where a more complex brand logo (including type and more structural elements) presented inside the three basic geometric shapes tested in this experiment, might give valuable insights in whether logo outlines can be recognized with the core logo to convey brand personality. With this study's support of marketing literature discussing logo angularity and roundness, further research into how these elements directly relate to brand personality could elucidate more substantial relationships between logo shape and brand personality. 



\section{Citations}

Aaker, J. L. (1997), “Dimensions of Brand Personality,” Journal of Marketing Research, 34 (3), 347-356.

Adîr, G., Adîr, V., Pascuc, N. E. (2012), "Logo Design and the Corporate Identity," Procedia Social and Behavioral Sciences, 51, 650-654.

Aguilar, A. G., et al. (2014), "Destination Brand Personality: An Application to Spanish Tourism," International Journal of Tourism Research, 18 (3) , 210-219.

Arhipova Alina (2018, Sept 6), "Knock Design into Shape. Psychology of Shapes,” Tubik Studio, Retrieved from www.tubikstudio.com

Aronoff, J. (2006), "How We Recognize Angry and Happy Emotion in People, Places, and Things," Cross-Cultural Research, 40 (1), 83-105.

Bar, M., \& Maital N. (2006), "Humans Prefer Curved Visual Objects,” Psychological Science, $17(8), 645-648$.

Bhavya M (2016, June 24), “The Geometry of Logo Designs,” Tech Story, Retrieved from www.techstory.in

"Brand as a beacon of change," 2018, Case study, Landor, Web, Accessed 17 November 2018

Broniarczyk, S. M., \& Alba J. W. (1994), "The Importance of the Brand in Brand Extension," Journal of Marketing Research, 31 (2), 214-228.

Cobb-Walgren, C. J., Ruble C. A. \& Donthu N. (1995), "Brand Equity, Brand Preference, and Purchase Intent," Journal of Advertising, 24 (3), 25-40.

Christie, M (2017, Sept 11), “The Psychology of Logo Shapes: A Designer's Guide,” Creative Bloq, Retrieved from www.creativebloq.com

Park, C. W., Eisingerich, A. B., Pol, G., \& Park, J. W. (2013), "The role of brand logos in firm performance," Journal of Business Research, 66 (2), 180-187.

Park, C. W., Jaworski, B. J. \& Maclnnis, D. J. (1986), "Strategic Brand Concept-Image Management," Journal of Marketing, 50 (4), 135-145. 
Dondis, D. A. (1973), A Primer of Visual Literacy, Cambridge, MA: MIT Press

Ekinci, Y., \& Hosany S. (2006), "Destination Personality: An Application of Brand Personality to Tourism Destinations," Journal of Travel Research, 45, 127-139

Farhana, M. (2012), "Brand Elements Lead to Brand Equity: Differentiate or Die," Information Management and Business Review, 4 (4), 223-233.

Fennis, B. \& Pruyn, H. (2007), "You are what you wear: Brand personality influences on consumer impression formation," Journal of Business Research, 60 (6), 634-639

Foroudi, P., et al. (2014), "Linking Corporate Logo, Corporate Image, and Reputation: An Examination of Consumer Perceptions in the Financial Setting," Journal of Business Research, 67 (11), 2269-2281.

Freling, T. H., Crosno, J. L. \& Henard, D. H. (2011), "Brand personality appeal: conceptualization and empirical validation," Journal of the Academy of Marketing Science. 39 (3). 392-406.

Frutiger, A, \& Andrew B. (1998), Signs and Symbols: Their Design and Meaning, Ebury Press, 1998.

Grinsven, B. V., \& Enny D. (2014), "Logo Design in Marketing Communications: Brand Logo Complexity Moderates Exposure Effects on Brand Recognition and Brand Attitude," Journal of Marketing Communications, 22 (3), 256-270.

Grohmann, B. (2008), "The Effect of Logo Design on Brand Personality Perceptions," Halifax, Nova Scotia.

Hayes, B., Alford, B.L., Silver, L. \& York, R.P. (2006), "Looks matter in developing consumer-brand relationships," Journal of Product \& Brand Management, 15 (5), 306-15.

Henderson, P. W., \& Cote J. A. (1998), "Guidelines for Selecting or Modifying Logos," Journal of Marketing, 62 (2), 14-30.

Hoeffler, S. \& Lane, K. K. (2003), "The Marketing Advantages of Strong Brands," Journal of Brand Management, 10 (6). 421-445. 
Faircloth, J. B., Capella, L. M. \& Alford, B. L. (2001), "The Effect of Brand Attitude and Brand Image on Brand Equity, Journal of Marketing Theory and Practice", 9 (3), 61-75.

"Geometry and Logo Design: What Shapes Say to Your Customers," (2017, Jan 29), The Logo Company, Retrieved from www.thelogocompany.net

Kay, M. (2006), "Strong Brands and Corporate Brands," European Journal of Marketing. 40 (7/8), 742-760.

Kim, C. K., Han, D. \& Park, S. (2001), "The effect of brand personality and brand identification on brand loyalty: Applying the theory of social identification," Japanese Psychological Research, 43 (4), 195-206.

Klink, R. R. (2003) "Creating Meaningful Brands: The Relationship Between Brand Name and Brand Mark," Marketing Letters, 14 (3), 143-57

Labrecque, L. I. \& Milne (2012), "Exciting Red and Competent Blue: The Importance of Color in Marketing," Journal of the Academy of Marketing Science. 40 (5), 711-727

Larson, C. L., et al. (2011), "Simple Geometric Shapes Are Implicitly Associated with Affective Value," Motivation and Emotion, 36 (3), 404-413

Luffarelli, J., Stamatogiannakis, A., Yang, H. (2019), “The Visual Asymmetry Effect: An Interplay of Logo Design and Brand Personality on Brand Equity," Journal of Marketing Research, 56 (1), 1-15.

Macnab, M. (2015, June 23), "How Geometry Influences Logo Design,” Smashing Magazine, Retrieved from www.smashingmagazine.com

Maehle, N., Otnes, C., \& Supphellen, M. (2011), "Consumers' perceptions of the dimensions of brand personality," Journal of Consumer Behaviour, 10 (5), 290-303.

Malär, L., Krohmer, H., Hoyer, W. D., \& Nyffenegger, B. (2011), "Emotional brand attachment and brand personality: The relative importance of the actual and ideal self," Journal of Marketing, 75 (4), 35-52.

Müller, Brigitte, et al., "The Effects of Visual Rejuvenation through Brand Logos," Journal of Business Research, 66 (1), 82-88. 
Pahwa, A. (2018, Oct 29), "Psychology Of Shapes In Logo Design,” Feedough, Retrieved from www.feedough.com

Pittard N., Ewing M., \& Jevons C., (2007), "Aesthetic theory and logo design: examining consumer response to proportion across cultures," International Marketing Review, 24 (4), 457-473.

Peck, J., \& Barger, V. (2009),"In Search of a Surrogate For Touch: the Effect of Haptic Imagery on Psychological Ownership and Object Valuation," Advances in Consumer Research, 36, 127-130.

Pettersson, R. (2000), Graphic Symbols-Design And Meaning, Natural Vistas: Visual Literacy \& The World Around Us, In R.E. Griffin, W. J. Gibbs, \& V. S. Williams.

“The Psychology of Logo Shapes,” (2018, Nov 6), Tailor Brands, Retrieved from www.tailorbrands.com

"Shape." Merriam-Webster.com. Merriam-Webster, 2018. Web. 17 November 2018.

Walsh, M. F. (2005), “Consumer Response to Logo Shape Redesign: the Influence of Brand Commitment," Katz Graduate School of Business, PhD Dissertation

Walsh, M. F. Winterich, K. P., \& Vikas M. (2010), "Do logo redesigns help or hurt your brand? The role of brand commitment," Journal of Product \& Brand Management, 19 (2) 76-84.

Westerman S.J., et al. (2013). "The design of consumer packaging: Effects of manipulations of shape, orientation, and alignment of graphical forms on consumers' assessments," Food Quality and Preference, 27 (1), 8-17.

Jiang, Y., Gorn, G. J., Galli, M., Chattopadhyay, A. (2016), "Does Your Company Have the Right Logo? How and Why Circular- and Angular-Logo Shapes Influence Brand Attribute Judgments," Journal of Consumer Research, 42 (5), 709-726.

Zmuda N., et al. (2008). "What Went Into the Updated Pepsi Logo.” Ad Age, Retrieved from adage.com/article/news/pepsi-s-logo-update/132016/. 


\section{Appendix}

Appendix A
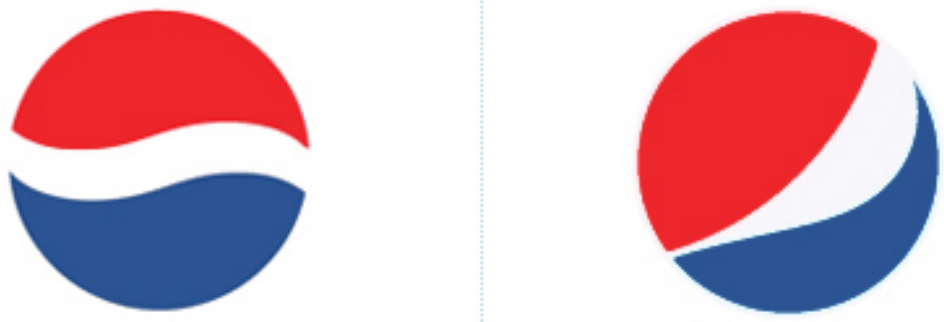

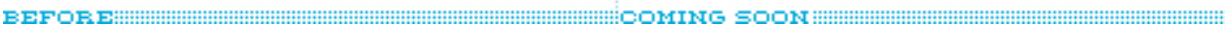

Retrieved from www.underconsideration.com

\section{Appendix B}
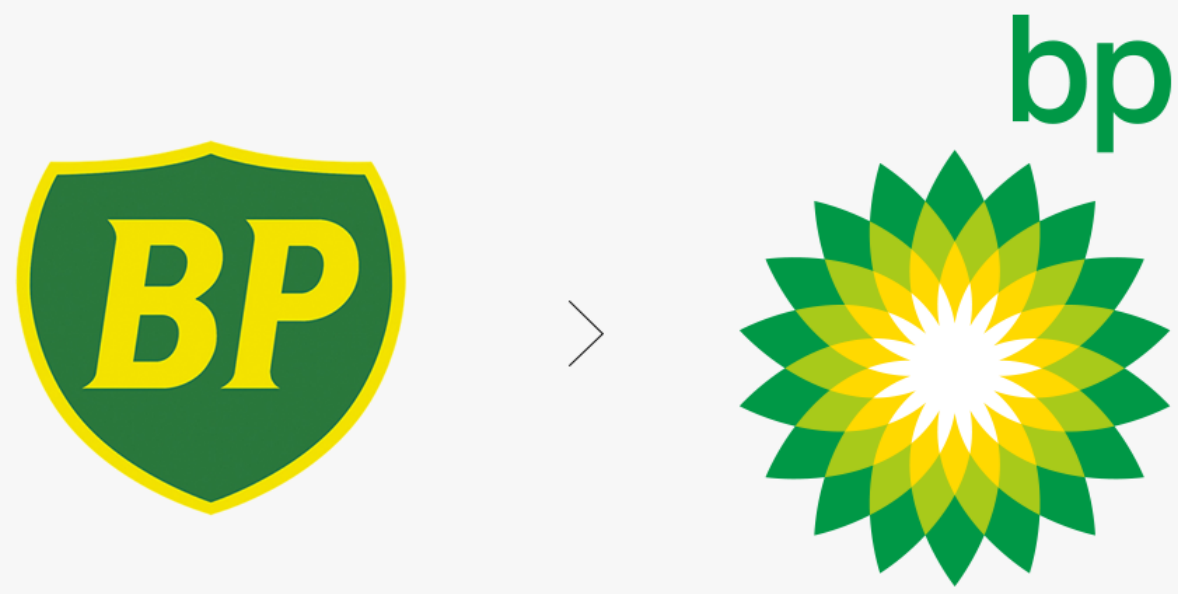

Retrieved from www.Landor.com 
Appendix C

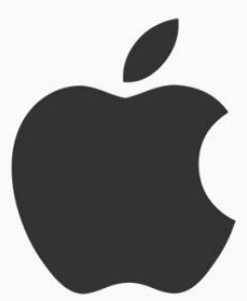

Retrieved from www.pcbuildersclub.com

Appendix D
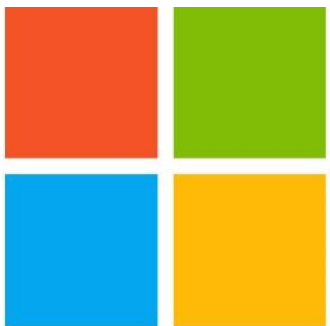

Retrieved from www.pcworld.com

\section{Appendix E}

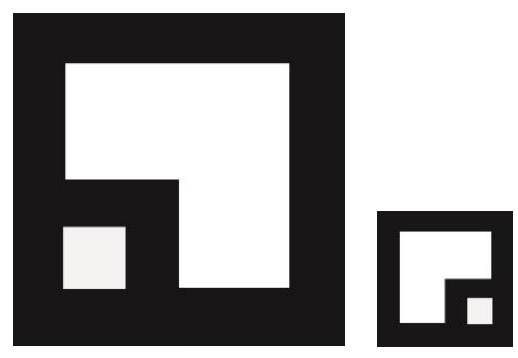

Appendix F

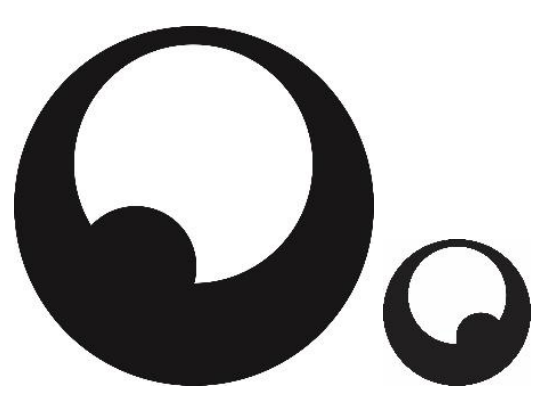




\section{Appendix G}

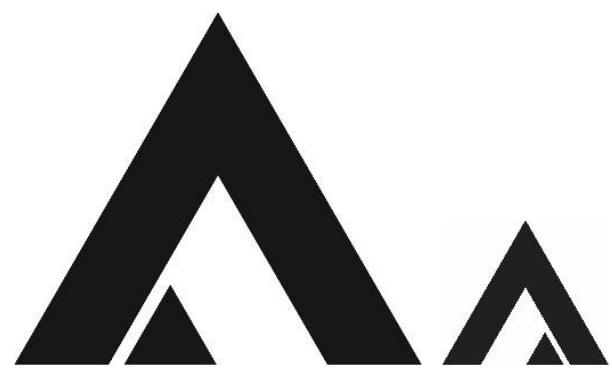

\section{Appendix $\mathrm{H}$}

\begin{tabular}{|l|r|r|r|r|r|r|}
\hline & Interesting & Quality & Good & Distinctive & Like \\
\hline Interesting & 1 & & & & \\
\hline Quality & 0.6793043 & 1 & & & \\
\hline Good & 0.7076817 & 0.74945 & 1 & & \\
\hline Distinctive & 0.5772784 & 0.5368 & 0.61262 & 1 & \\
\hline Like & 0.6405402 & 0.65268 & 0.80999 & 0.6033 & 1 \\
\hline
\end{tabular}

\section{Appendix I}

\begin{tabular}{|c|c|c|c|}
\hline & Square & Circle & Triangle \\
\hline Square & 1 & -0.5 & -0.5 \\
\hline Square & & $<.0001$ & $<.0001$ \\
\hline Circle & -0.5 & 1 & -0.5 \\
\hline Circle & $<.0001$ & & $<.0001$ \\
\hline Triangle & -0.5 & -0.5 & 1 \\
\hline Triangle & $<.0001$ & $<.0001$ & \\
\hline \multirow[t]{2}{*}{ affect } & -0.32149 & 0.09011 & 0.23138 \\
\hline & $<.0001$ & 0.1513 & 0.0002 \\
\hline Down_to_earth & -0.18173 & 0 & 0.18173 \\
\hline Down-to-earth & 0.0036 & 1 & 0.0036 \\
\hline Honest & -0.1305 & 0.0752 & 0.0553 \\
\hline Honest & 0.0373 & 0.2314 & 0.3792 \\
\hline Wholesome & -0.19685 & 0.18279 & 0.01406 \\
\hline Wholesome & 0.0016 & 0.0034 & 0.8232 \\
\hline Cheerful & -0.24398 & 0.19104 & 0.05294 \\
\hline Cheerful & $<.0001$ & 0.0022 & 0.3999 \\
\hline Daring & -0.20824 & -0.0359 & 0.24414 \\
\hline Daring & 0.0008 & 0.5682 & $<.0001$ \\
\hline Spirited & -0.31954 & 0.15647 & 0.16308 \\
\hline Spirited & $<.0001$ & 0.0124 & 0.0091 \\
\hline Imaginative & -0.2369 & 0.21769 & 0.01921 \\
\hline Imaginative & 0.0001 & 0.0005 & 0.7602 \\
\hline Up_to_date & 0.01422 & -0.06401 & 0.04978 \\
\hline Up-to-date & 0.8212 & 0.3086 & 0.4286 \\
\hline Reliable & 0.05998 & -0.14073 & 0.08075 \\
\hline Reliable & 0.3401 & 0.0246 & 0.1987 \\
\hline Intelligent & 0.09388 & -0.04023 & -0.05364 \\
\hline Intelligent & 0.1349 & 0.5224 & 0.3936 \\
\hline Successful & 0.03918 & -0.12674 & 0.08757 \\
\hline Successful & 0.5335 & 0.0432 & 0.1633 \\
\hline Upperclass & 0.00487 & -0.08271 & 0.07784 \\
\hline Upperclass & 0.9384 & 0.188 & 0.2154 \\
\hline Charming & -0.20008 & 0.18056 & 0.01952 \\
\hline Charming & 0.0013 & 0.0038 & 0.7564 \\
\hline Outdoorsy & -0.27864 & -0.19384 & 0.47248 \\
\hline Outdoorsy & $<.0001$ & 0.0019 & $<.0001$ \\
\hline Tough & -0.00902 & -0.32005 & 0.32907 \\
\hline Tough & 0.8861 & $<.0001$ & $<.0001$ \\
\hline
\end{tabular}

